\title{
The Role of PEG-40-stearate in the Production, Morphology, and Stability of Microbubbles
}

Joshua Owen, ${ }^{\dagger}$ Sukanta Kamila, ${ }^{\ddagger}$ Shamit Shrivastava, ${ }^{\dagger}$ Dario Carugo, ${ }^{\S}$ Jorge Bernardino de la Serna," Christophoros Mannaris,,$^{\dagger}$ Valerio Pereno, ${ }^{\dagger}$ Richard Browning, ${ }^{\dagger}$ Estelle Beguin, ${ }^{\dagger}$ Anthony P. McHale, ${ }^{\dagger}$ John F. Callan, ${ }^{,}$and Eleanor Stride ${ }^{* \dagger \odot}$

${ }^{\dagger}$ Old Road Campus Research Building, University of Oxford, Oxford OX3 7DQ United Kingdom

${ }^{\ddagger}$ School of Pharmacy and Pharmaceutical Science, University of Ulster, Coleraine BT52 1SA, United Kingdom

${ }^{\S}$ Faculty of Engineering and the Environment, University of Southampton, Highfield, Southampton SO17 1BJ, United Kingdom

"Central Laser Facility, STFC Rutherford Appleton Laboratory, Harwell Campus, Didcot OX11 0QX, United Kingdom

Supporting Information

ABSTRACT: Phospholipid coated microbubbles are currently in widespread clinical use as ultrasound contrast agents and under investigation for therapeutic applications. Previous studies have demonstrated the importance of the coating nanostructure in determining microbubble stability and its dependence upon both composition and processing method. While the influence of different phospholipids has been widely investigated, the role of other constituents such as emulsifiers has received comparatively little attention. Herein, we present an examination of the impact of polyethylene glycol (PEG) derivatives upon microbubble structure and properties. We present data using both pegylated phospholipids and a fluorescent PEG-40-stearate analogue synthesized inhouse to directly observe its distribution in the microbubble coating. We examined microbubbles of clinically relevant sizes, investigating both their surface properties and population size distribution and stability. Domain formation was observed only on the surface of larger microbubbles, which were found to contain a higher concentration of PEG-40-stearate. Lipid analogue dyes were also found to influence domain formation

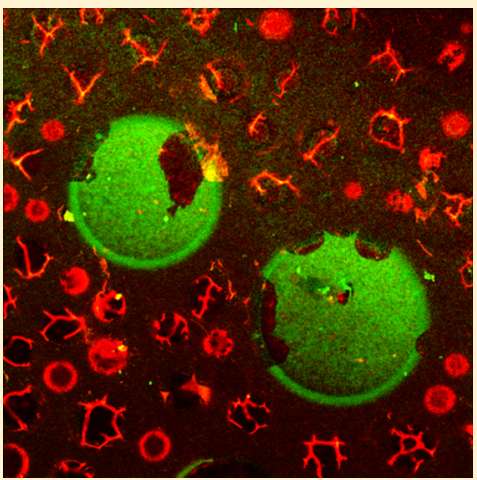
compared with PEG-40-stearate alone. "Squeezing out" of PEG-40-stearate was not observed from any of the microbubble sizes investigated. At ambient temperature, microbubbles formulated with DSPE-PEG(2000) were found to be more stable than those containing PEG-40-stearate. At $37^{\circ} \mathrm{C}$, however, the stability in serum was found to be the same for both formulations, and no difference in acoustic backscatter was detected. This could potentially reduce the cost of PEGylated microbubbles and facilitate simpler attachment of targeting or therapeutic species. However, whether PEG-40-stearate sufficiently shields microbubbles to inhibit physiological clearance mechanisms still requires investigation.

\section{INTRODUCTION}

Ultrasound offers a safe, convenient, and cost-effective method of medical diagnostic imaging. Image contrast between blood vessels and the surrounding tissue, however, is typically lower than in other modalities such as magnetic resonance imaging (MRI). ${ }^{1}$ To overcome this limitation, ultrasound contrast agents consisting of gas microbubbles stabilized by a surfactant or polymer coating ${ }^{2}$ are injected into the patient's bloodstream. Due to their high compressibility, microbubbles are able to scatter ultrasound much more efficiently than red blood cells. Moreover, they exhibit a nonlinear response enabling the scattered signal to be distinguished from that due to neighboring tissues. ${ }^{1}$ Therapeutically, the oscillations of microbubbles under ultrasound can increase the permeability of both cell and tissue membranes for delivery of bioactive compounds. ${ }^{3}$ Microbubbles are also being explored for use as oxygen carriers for the treatment of ischemia and tumor hypoxia. $^{4-6}$
The key component of the microbubble for imparting stability and preventing coalescence is the coating. Microbubbles formulated with cross-linked serum albumin, such as Albunex and Optison, have relatively high coating stiffness and the propensity to rupture beyond a critical strain/expansion ratio, which limits the duration of contrast enhancement. To overcome this, Levovist used a surfactant (palmitic acid) to provide a more flexible coating and hence improve the acoustic response, but this resulted in a reduction in stability. Subsequently, however, it was found that other surfactants such as phospholipids could simultaneously provide stability and a strong acoustic response. This has led to multiple

Special Issue: Microbubbles: Exploring Gas-Liquid Interfaces for Biomedical Applications

Received: July 25, 2018

Revised: November 26, 2018

Published: November 28, 2018 
phospholipid formulations reaching the clinic, in particular Sonovue (Bracco Imaging), Sonazoid (GE Healthcare) and Definity (Bristol Myers-Squibb). In addition to phospholipids, smaller quantities of an emulsifier, typically polyethylene glycol derivatives are used in microbubble formulations to promote bubble formation, inhibit coalescence, and reduce nonspecific adsorption of blood plasma proteins.

Only a limited number of studies have been performed that examine the composition and structural properties of the phospholipid microbubble coating. For instance, Kim et al. analyzed the mechanical properties of phospholipid monolayers using micropipette aspiration. They also employed fluorescent phospholipids and freeze fracture electron micros$\operatorname{copy}^{8}$ to examine domain formation in the coating. This study utilized microbubbles composed of 10:1 lipid:PEG-40-stearate molar ratio and varied both lipid chain length and cooling time during bubble fabrication. The results indicated that the monolayer microstructure was dependent on the cooling rate with larger domains formed at slower cooling rates. Bubbles with a coarse domain structure exhibited a resistance to shear deformation higher than those with a fine structure.

Borden et al. examined the surface phase behavior and microstructure of lipid/PEG-emulsifier coated microbubbles again with lipids of different chain length and a fluorescent dye (NBD-PC) via fluorescence microscopy and using lipid films in a Langmuir trough. ${ }^{9}$ These results confirmed the polycrystalline nature of the two-component coating and that the surface morphology was affected by the bubble fabrication process. NMR and FTIR spectroscopy were then employed to investigate lateral phase separation. These results indicated that, at least in microbubbles greater than $10 \mu \mathrm{m}$ in diameter, the coating comprised ordered domains consisting of lipid and interdomain regions enriched by PEG-40-stearate. ${ }^{10}$ It was also suggested that below a certain size $(<20 \mu \mathrm{m})$, the bubble surface pressure would be too high for the emulsifier rich regions to coexist with the lipid domains and would only remain attached through surface associated aggregates.

Lozano and Longo ${ }^{11}$ employed fluorescence microscopy to study dissolving microbubbles coated with either a phosphatidylcholine (C16, C18, or C20) and PEG-40-stearate mixture or a phosphatidylcholine (C16, C18, or C20) and a PEG conjugated phospholipid (DSPE-PEG(2000)). Dissolution times for microbubbles containing DSPE-PEG(2000) were four times longer than those containing PEG-40-stearate. This is because the DSPE-PEG(2000) remains in the condensed phase with the lipid, whereas with PEG-40-stearate, it separates into the expanded phase, which is more gas permeable. Unlike the studies of Kim et al. ${ }^{8}$ and Borden et al. ${ }^{9}$, these experiments were performed with microbubbles in degassed water, using the lipid analogue DiIC18 fluorophore to examine domain formation and/or surface deformation. Moreover, these experiments were performed with air-filled bubbles (rather than the clinically used perfluorocarbons which have been shown to alter the properties of lipid membranes ${ }^{12-14}$ ) and at room temperature with bubbles of approximately $20 \mu \mathrm{m}$.

From these previous experiments, the behavior of the emulsifier was thus inferred from the observed lipid behavior. Moreover, the fluorescence-based technique for the observation of lipid domains was applied on microbubbles above $5 \mu \mathrm{m}$ only to enable resolution of surface structures.

Kooiman et al. ${ }^{15}$ investigated the impact of lipid chain length on the distribution of ligands around the microbubble surface, using super-resolution microscopy. Two lipid chain lengths,
DPPC (16 carbons) and DSPC (18 carbons), were used to make microbubbles with DSPE-PEG(2000)-biotin. Fluorescent streptavidin was attached to biotinylated microbubbles, and a more even distribution and larger targeting area was found with DPPC relative to DSPC. ${ }^{15}$ This was attributed to the fact that DSPE-PEG(2000)-biotin was more heterogeneously distributed on the microbubble surface in DSPC relative to DPPC, reducing the targeting area.

Abou-Saleh et al. examined the impact of PEGylated lipids on microbubbles created by microfluidics and observed changes in microbubble stability and mechanical properties with increasing concentration of PEGylated lipid. Above a certain threshold, the lifetime and concentration of the microbubbles were seen to decrease. ${ }^{16}$ Shih et al. performed similar experiments and found bubble lifetime to be related to stable size, and that bubble size reduced with increasing concentration of emulsifier, down to a threshold of $30 \%$ beyond which stability was compromised. ${ }^{17}$ Segers et al. also found that during insonation shedding of the pegylated lipid from the microbubble can occur potentially altering the coating stiffness. ${ }^{18}$

Recent results from Carugo et al. have shown transfer of material from the microbubble coating to nearby cell membranes and that polyethylene glycol has a major impact on lipid ordering and the extent of this lipid transfer. ${ }^{19}$ Hosny et al. used molecular rotor fluorescence lifetime imaging to quantify the spatial distribution of viscosity in a microbubble coating. Varying composition and production methodologies indicated that there can be a large variation in viscosity across a microbubble population independent of the individual microbubble size. ${ }^{20}$

The aim of this study is to build on the results of previous research examining the properties of microbubbles formulated with PEG-40-stearate. A fluorescent PEG-40-stearate analogue was synthesized to visualize the location of PEG-40-stearate in the microbubble coating. The impact of PEG-40-stearate concentration on the lipid packing was also examined via fluorescence spectral imaging of an environmentally sensitive fluorophore (C-laurdan). The concentration, size, and stability of microbubbles formulated with both PEG-40-stearate and DSPE-PEG(2000) were then measured. Only one chain length of 18 carbons (DSPC) was used throughout the experiments for consistency. Finally, the acoustic responses of the different microbubbles were compared.

\section{MATERIALS AND METHODS}

Materials. 1,2-Distearoyl-sn-glycero-3-phosphocholine (DSPC) was purchased from Avanti Polar Lipids Inc. (Alabaster, Alabama, United States). PEG-40-stearate, ethanol, chloroform, PEG (2000), stearoyl chloride, and human serum were purchased from SigmaAldrich Ltd. (Gillingham, Dorset, UK). C-Laurdan was received as a kind gift from Dr. Erdinc Sezgin (Weatherall Institute of Molecular Medicine, University of Oxford, Oxford, UK) but can be purchased from Stratech Scientific Ltd., Cambridge House, St. Thomas' Pl, Ely. PEG (2000) FITC and fluorescein-PEG-NH $\mathrm{NH}_{2}(\mathrm{MW}=2000)$ were purchased from NANOCS (ChemQuest, Co., Ltd., Springfield House, Water Lane, Wilmslow, Cheshire, SK9 5BG, UK). Dil Stain (1,1'-dioctadecyl-3,3,3',3'-tetramethylindocarbocyanine perchlorate) was purchased from Thermo Fisher Scientific Ltd., United States.

Microbubble and Vesicle Preparation. The relevant mixtures of lipids and emulsifier, dissolved in chloroform, were mixed in a glass vial at a selected molar ratio (9:1) lipid to emulsifier. ${ }^{9,11,20}$ This was heated to $50{ }^{\circ} \mathrm{C}$ and left for $12 \mathrm{~h}$. The lipid film was suspended in aqueous solution $(2 \mathrm{~mL})$ for over $1 \mathrm{~h}$ at $75^{\circ} \mathrm{C}$ under constant stirring. The stir bar was removed, and the solution was then sonicated for 90 
$\mathrm{s}$ using an ultrasonic cell disruptor (XL2000, probe diameter $3 \mathrm{~mm}$; Misonix Inc., Farmingdale, NY, United States) operating at $22.5 \mathrm{kHz}$ and level 4 corresponding to $8 \mathrm{~W}_{\mathrm{RMS}}$ output power. Sonication at the gas-water interface in an $\mathrm{SF}_{6}$ gas environment was then performed for $20 \mathrm{~s}$ at sonication level $19\left(38 \mathrm{~W}_{\mathrm{RMS}}\right)$. The microbubble suspension was then placed in an ice bath for cooling for approximately $10 \mathrm{~min}$. The same procedure was followed for vesicles, but the last sonication step was omitted.

For those experiments involving fluorescent labeling of microbubbles, a stock solution of the lipophilic dye DiI at a concentration of $2.5 \mathrm{mg} / \mathrm{mL}$ in dimethyl sulfoxide (DMSO, Sigma-Aldrich, UK) was prepared. Seven microliters of the stock solution was added to the chloroform lipid solution, and the protocols outlined previously were followed for microbubble production. To remove excess DiI from the formed microbubble suspension, the microbubble sample was centrifuged once at $1000 \mathrm{~g}$ for $10 \mathrm{~min}$ (400R Heraeus Labofuge, Thermo Fisher Scientific Inc., United States), and microbubbles were resuspended in PBS after each centrifugation cycle.

Synthesis of Fluorescein Labeled PEG-40-Stearate. Fluorescein-PEG-stearate was synthesized according to Scheme 1.

\section{Scheme 1. Synthesis of Fluorescein-PEG-stearate}
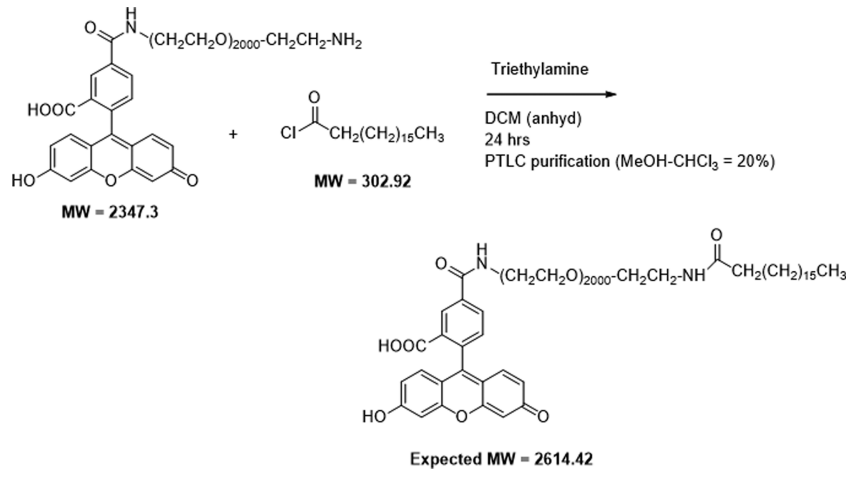

To an ice cold solution $\left(5{ }^{\circ} \mathrm{C}\right)$ of fluorescein-PEG- $\mathrm{NH}_{2}(50 \mathrm{mg}$, $0.02 \mathrm{mmol})$ in anhydrous dichloromethane $(5 \mathrm{~mL})$ under $\mathrm{N}_{2}$ atmosphere was added a catalytic amount of triethylamine. The solution was stirred for $30 \mathrm{~min}$ at $5{ }^{\circ} \mathrm{C}$, and a solution of stearoyl chloride $(7 \mathrm{mg}, 0.02 \mathrm{mmol})$ in anhydrous dichloromethane $(5 \mathrm{~mL})$ was added dropwise, maintaining the temperature between 0 and 5 ${ }^{\circ} \mathrm{C}$. The reaction was allowed to proceed at room temperature for 24 $\mathrm{h}$ and kept in the dark. The product fluorescein-PEG-stearate was recovered by dissolution in dichloromethane and precipitation in ether and finally purified by preparative thin layer chromatography using methanol-chloroform (20\%) as the eluant.

Measurement of Lipid Order and Lipid Structure in the Microbubble Coating. C-Laurdan Staining. The lateral order of the lipids within the microbubble coating was quantified using Claurdan, an environmentally sensitive fluorescent probe, the emissions from which shift according to the degree of hydration in the membrane. This technique has been widely used to characterize the surface properties of lipid structures, including investigation of the interactions between microbubbles and cells. ${ }^{19}$ To reduce fluorescence background due to lipids in suspension, microbubbles were washed once by centrifugation. Approximately $3 \mathrm{~mL}$ of freshly prepared microbubbles were loaded into a disposable $10 \mathrm{~mL}$ LuerLok syringe (BD, MediSupplies, Unit 3, 2 Lansdowne Crescent, Bournemouth, UK) adapted to fit upright within a $50 \mathrm{~mL}$ centrifuge tube (Corning, Fogostraat 12, 1060 LJ Amsterdam, The Netherlands). They were then centrifuged at $1000 \mathrm{~g}$ relative centrifugal force (RCF) at $10{ }^{\circ} \mathrm{C}$ for $10 \mathrm{~min}$ in a swing bucket rotor (Heraeus Labofuge 400R, Thermo Fisher Scientific, Waltham, MA, United States) to form a bubble "cake" against the plunger. The subnatant was discarded, and the bubbles were resuspended within the syringe using $2 \mathrm{~mL}$ of PBS. This was then transferred to a glass vial for future use. To stain with C-laurdan, $1.5-5 \mu \mathrm{L}$ of C-laurdan $(400 \mathrm{nM})$ was added to $30 \mu \mathrm{L}$ of washed microbubbles diluted in $70 \mu \mathrm{L}$ of Milli-Q water (total sample volume $=100 \mu \mathrm{L}$ ). This was incubated for up to $30 \mathrm{~min}$ at $4{ }^{\circ} \mathrm{C}$ before use.

Spectral Fluorescence Imaging. Spectral imaging was performed on a LSM 780 Inverted Confocal Microscope (Carl Zeiss Microscopy $\mathrm{GmbH}$, Jena, Germany) using a previously reported methodology. ${ }^{21}$ Briefly, $10 \mu \mathrm{L}$ of stained microbubbles was loaded onto a $170 \mu \mathrm{m}$ thick glass slide coverslip (Logitech Ltd., Scotland) and covered with another coverslip. Individual microbubbles were then located and imaged using an oil immersed $63 \times$ objective with the microscope focus set at the midplane of the microbubble. Spectral imaging was performed using a Zeiss LSM 780 confocal microscope (Carl Zeiss Microscopy GmbH, Jena, Germany) equipped with a 32-channel GaAsP detector array. C-Laurdan was excited at $405 \mathrm{~nm}$, and the lambda detection range was set between 415 and $691 \mathrm{~nm}$. This resulted in a single image stack with each image providing the intensity map at a single emission wavelength $(n=30)$. Approximately 20 images were taken per formulation, and each formulation was repeated three times using a freshly prepared microbubble suspension created from a new lipid film.

Generalized Polarization (GP) Calculation. GP is an optical observable that quantifies the shift in the emission spectra of the dye. It provides an order parameter for the phase transition discussed above and is related to the dielectric properties of its microenvironment, which in turn is directly affected by the presence of water in the lipid film. For example, an increase in hydration causes a red shift in the spectrum, which corresponds an increase in GP values. GP (ranging from -1 to +1 ) was utilized as a relative measure of lipid packing within the microbubble shell. To measure this, fluorescence microscopy images were processed using a custom script in MATLAB (The Mathworks Inc.). Briefly, images were first opened using the BioFormats for MATLAB script. Microbubbles were identified by a semiautomated implementation of the imfindcircles () function, and the background and internal volume of the bubble were removed by cropping, leaving only the pixels of the stained bubble coating. ${ }^{21}$ This was done to reduce anisotropy effects and out-of-focal plane effects of the coating. GP values were calculated (per pixel) using intensity data from the 440 and $490 \mathrm{~nm}$ wavelength stacks by the following equation: ${ }^{22}$

$$
\mathrm{GP}=\frac{I_{440}-I_{490}}{I_{440}+I_{490}}
$$

Increased GP value corresponds to an increase in the lipid order of the coating layer, and vice versa. Whole microbubble GP values were calculated from the average of the GP per pixel values. Standard deviations, medians, and histogram plots were also generated from these data. Images with saturation (intensity of 255) in any wavelength were discarded. For spectral analysis, average intensity values of the coating per wavelength were calculated from pixel intensity values. ${ }^{20}$

Lipid Domain Analysis by Langmuir Trough. Lipids from commercial stocks were used without further purification and were mixed in chloroform to obtain desired ratios at $25 \mathrm{mg} / \mathrm{mL}$. Two microliters was then spread at the air-water interface in a Langmuir trough (microtrough G2, Kibron, Malminkaari, Helsinki, Finland) with an initial area of $212 \mathrm{~cm}^{2}$. The trough was compressed at 50 $\mathrm{cm}^{2} / \mathrm{min}$, and the pressure was recorded using a Wilhelmy plate at room temperature. Fluorescence images of the DSPC:PEG-40stearate monolayer were obtained by titrating the lipids under a microscope in $100 \mathrm{~mm}$ diameter Petri dish. The amount of lipids to be titrated was estimated from the isotherm on the Langmuir trough.

Fluorescence Microscopy of Microbubbles. The stained microbubbles were pipetted onto a $1.5 \mathrm{H} 75 \times 25 \mathrm{~mm}$ glass slide, and a coverslip was placed to prevent movement of the sample. A Zeiss LSM 780 confocal microscope equipped with a $63 \times$ PlanApochromat objective was used for imaging the microbubbles in the equatorial plane. The pinhole was adjusted to modify the thickness of the imaging plane and capture the phase separation on the microbubble surface. The imaging was performed on two separate 

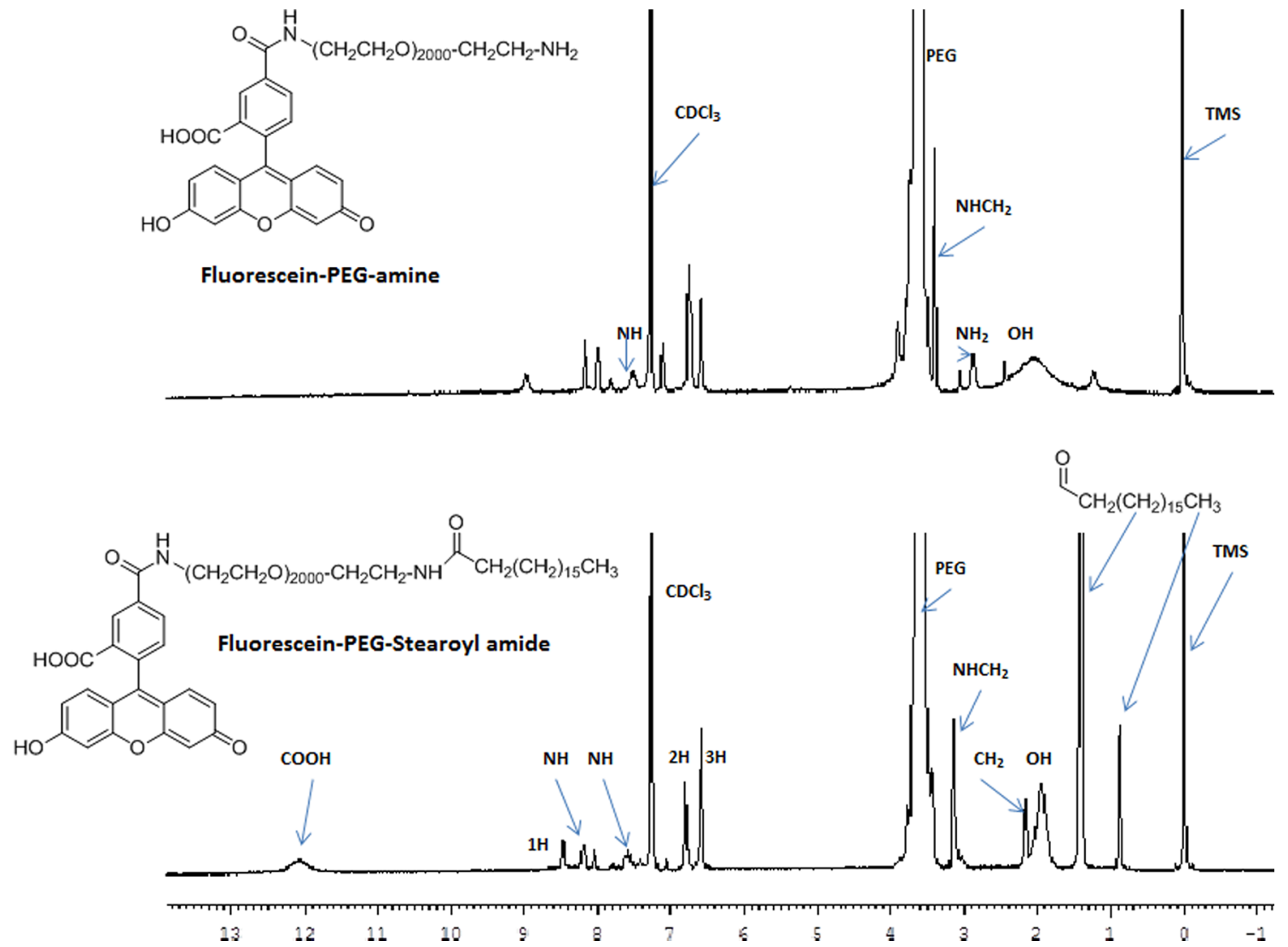

Figure 1. ${ }^{1} \mathrm{H}$ NMR spectra of fluorescein-PEG and fluorescein-PEG-stearate (solvent: $\mathrm{CDCl}_{3}$ ).

channels: the first involved a $543 \mathrm{~nm}$ excitation and detectors set to a 553-673 nm range for detection of DiI; the second consisted of a 488 $\mathrm{nm}$ excitation with detectors set to acquire at $493-553 \mathrm{~nm}$ for detection of FITC. Intensity/pixel was determined using ImageJ public domain software (NIH, Bethesda, MD) by selecting the total area of the microbubble shell in midplane and measuring the raw intensity and the number of pixels.

Structure of Fluorescent PEG-40-stearate Analogue. Mass Spectroscopy Determination of Molecular Weight. Matrix-assisted laser desorption/ionization-time-of-flight (MALDI-TOF) spectra of fluorescein-PEG-stearate (Figure 1) was performed in Voyager-DMBio spectrometer using $50 \mu \mathrm{L}$ of $1 \mathrm{mg} / 3 \mathrm{~mL}$ sample mixed with 100 $\mu \mathrm{L}$ of DHB matrix, where the laser intensity was 1471 . The numberaverage molecular weight $\left(M_{n}\right)$ and average molecular weight $\left(M_{w}\right)$ were calculated by the following equations:

$$
\begin{aligned}
& M_{\mathrm{n}}=\sum N_{\mathrm{i}} M_{\mathrm{i}} / \sum N_{\mathrm{i}} \\
& M_{\mathrm{w}}=\sum N_{\mathrm{i}} \mathrm{M}_{\mathrm{i}}^{2} / \sum N_{\mathrm{i}} M_{\mathrm{i}}
\end{aligned}
$$

where $M_{\mathrm{i}}$ is the molecular weight of the chain and $N_{i}$ is the number of chains of that molecular weight.

${ }^{1} \mathrm{H}$ NMR Spectra Determination of Molecular Structure. To determine the structure and confirm the synthesis had been successful, the ${ }^{1} \mathrm{H}$ NMR spectrum of fluorescein-PEG-stearate was recorded at room temperature using a Varian spectrometer operating at $500 \mathrm{MHz}$ by using $\mathrm{CDCl}_{3}$ as solvent. The relative frequency of a nucleus relative to a standard in a magnetic field or chemical shifts $(\delta)$ are given in parts per million (ppm) using tetramethylsilane (TMS) as an internal reference.

Microbubble Characterization. Microbubble Size and Stability. Microbubble suspension $(10 \mu \mathrm{L})$ was added to a Neubauer hemocytometer (Sigma-Aldrich Ltd. Gillingham, Dorset, UK). This was then imaged using a Leica DM500 optical microscope (Larch House, Milton Keynes, MK14 6FG) and a 40× objective lens at room temperature. To obtain a representative size distribution for one batch of bubbles, at least 3 separate bubble samples must have 30 images taken via optical microscopy. ${ }^{23}$ The bubble size distribution and concentration were then determined using a purpose written image analysis software in Matlab (2013b, The Mathworks, Natick, MA, United States). The software converts each micrograph to a binary image, and single spheroid shapes are then detected, measured and counted for each of the images analyzed. A size distribution and count are then produced for the microbubbles. Because the dimensions of the hemocytometer and the microscope field of view are known, the volume concentration of microbubbles can also be estimated.

Nanoparticle Size Measurement. To determine the presence and size of vesicles, measurements of lipid dispersions before sonication at the gas-water interface were performed via dynamic light scattering using a Zetasizer Nano-ZS, Malvern Instruments Ltd. (Worcestershire, UK) in a disposable capillary cell (DTS1070 Malvern Instruments Ltd., Worcestershire, UK). Each sample of the liposome or microbubble solution $(60 \mu \mathrm{L})$ was added to $940 \mu \mathrm{L}$ of $10 \%$ HEPES buffer.

Microparticle Size Measurement. Laser light obscuration and scattering of microbubbles was used to obtain accurate size and concentration using an Accusizer 780, NICOMP Particle Sizing System (Santa Barbara, CA). Samples $(10 \mu \mathrm{L})$ of each microbubble suspension were diluted into a $50 \mathrm{~mL}$ flask under mild mixing during measurement.

Microbubble Stability in Serum. DSPC microbubbles at 9:1 molar ratio with PEG-40-stearate or DSPE-PEG-2000 and a set total concentration of $3 \mathrm{mg} / \mathrm{mL}$ were created, kept on ice, and added to serum. The microbubble size was then measured via the Accusizer. Ten microliters of the microbubble suspension was added to $500 \mu \mathrm{L}$ of serum, giving a concentration of $10^{7}$ microbubbles $/ \mathrm{mL}$. This is $10 \times$ the blood concentration of microbubbles in vivo, ${ }^{24}$ but was used as lower concentrations could not be detected. The Accusizer was calibrated to remove the contribution of particles in the serum. The change in microbubble concentration was then measured over time 


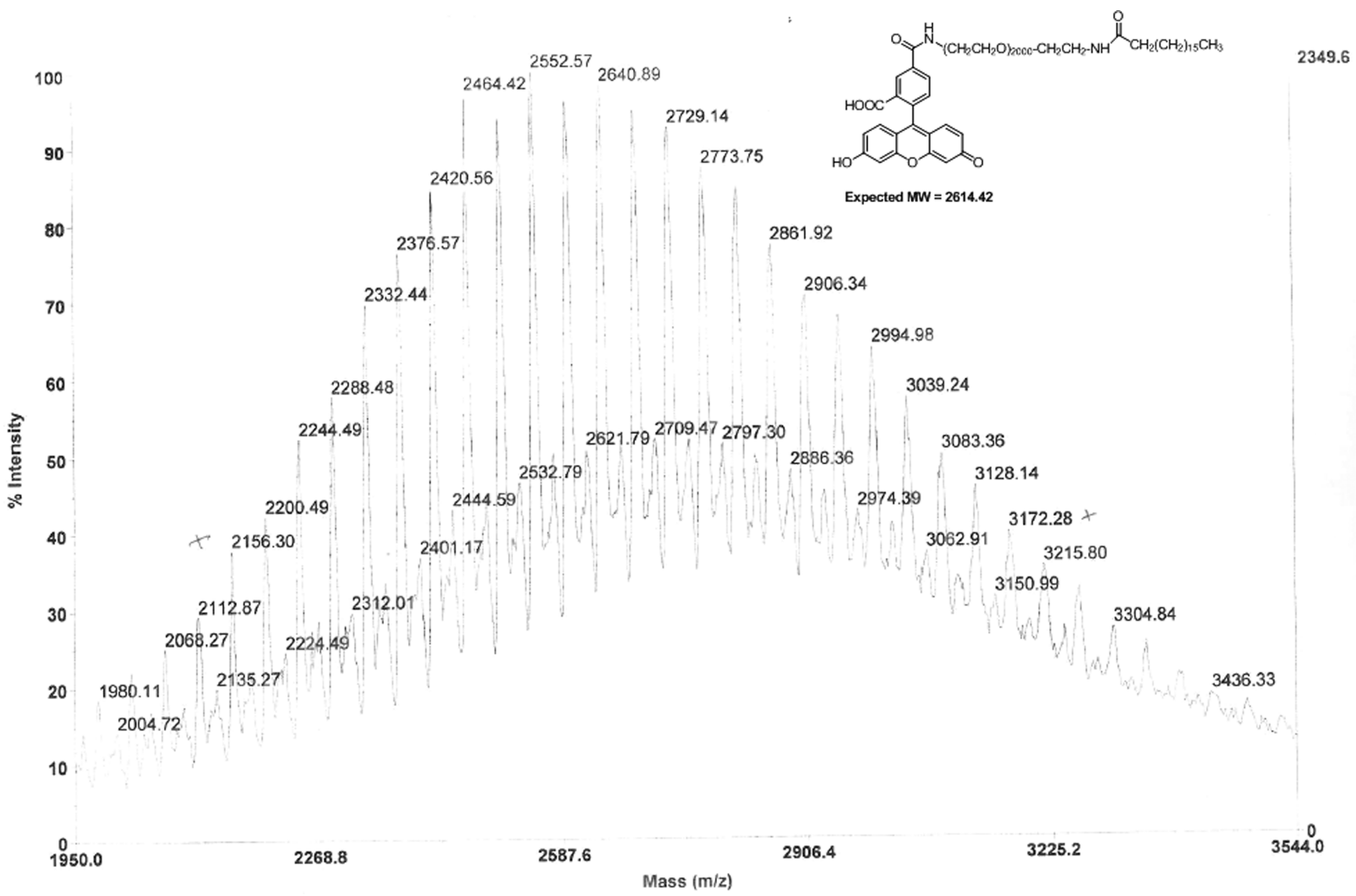

Figure 2. MALDI-TOF spectra of fluorescein-PEG-stearate amide indicating that stearic acid had been attached to the fluorescent PEG chain.

for $15 \mathrm{~min}$ (the typical half-life of microbubbles in the human body being less than $5 \mathrm{~min}){ }^{25}$ This was performed at both room temperature and $37^{\circ} \mathrm{C}$.

Measurement of Backscatter Acoustic Intensity of Microbubbles. Imaging of the microbubbles and measurement of their response to ultrasound was carried out using a tissue-mimicking agarose flow phantom model with an embedded $1.2 \mathrm{~mm}$ channel. ${ }^{26}$ A peristaltic pump (Minipuls Evolution, Gilson Scientific UK, Dunstable, Bedfordshire) was used to flow the freshly prepared microbubble solution $(500 \mu \mathrm{L})$ through the channel at a flow rate of $2 \mathrm{~mL} / \mathrm{min}$. An L12-5 probe of the iU-22 diagnostic ultrasound scanner (Philips Medical Systems, Bothell, WA) was used to interrogate and image the microbubbles at contrast mode and a low nondestructive mechanical index $(\mathrm{MI}=0.04)$. The frame rate was set at $1 \mathrm{~Hz}$ to ensure replenishment of the channel with fresh microbubbles, and video loops of at least 10 images were captured. Quantification of the average backscatter intensity was done in Matlab (Mathworks, Natick MA, United States). The procedure was repeated for three separate solutions for each type of microbubble. Experiments were carried out in a degassed water tank at $37^{\circ} \mathrm{C}$. Statistical analysis was performed using a one-tailed Student's $t$-test.

\section{RESULTS AND DISCUSSION}

Characterization of Fluorescein-PEG-stearate. Successful formation of the fluorescein-PEG-stearate was confirmed by ${ }^{1} \mathrm{H}$ NMR spectroscopy with Figure 1 showing the stacked spectra of the starting material fluorescein-PEG- $\mathrm{NH}_{2}$ and the product fluorescein-PEG-stearate. Resonances in the downfield region (i.e., between 6.0 and $8.0 \mathrm{ppm}$ ) were attributed to the aromatic protons of the fluorescein ring and were largely unchanged in the spectrum of the product, given their distance from the site of conjugation. Examination of the upfield region, however, revealed the appearance of three new resonances in the spectrum of the product at $0.88,1.40$, and $2.10 \mathrm{ppm}$ that were not present in the spectrum of the starting material and represent the methyl protons, the protons from the 15 methylene groups of the stearic acid aliphatic chain, and the methylene protons adjacent to newly formed amide carbonyl group, respectively. The broad peak at $3.9 \mathrm{ppm}$ in both spectra was characteristic of methylene protons in the PEG polymer chain, and again, their chemical shift remained unchanged in the product when compared to the starting material. This was expected as their proximity to the newly created amide bond was separated by the two methylene groups, minimizing any change to their electronic environment following conjugation. The ratio of the integrals for the new methyl protons and the eight aromatic protons was also consistent with successful product confirmation.

The molecular weight of fluorescein-PEG-stearate was also analyzed by MALDI-TOF mass spectroscopy (Figure 2). Given the polymeric nature of the PEG component of fluorescein-PEG-stearate, it is difficult to identify an exact molecular weight for this compound. However, assuming the total repeat unit weight was exactly $2000 \mathrm{Da}$, the MW would be expected to be $2614 \mathrm{Da}$. Similarly, the fluorescein-PEG$\mathrm{NH}_{2}$ precursor would be expected to have a MW $=2347 \mathrm{Da}$. Analysis of the MALDI-TOF spectrum revealed the fluorescein-PEG-stearate had a $M_{\mathrm{n}}$ and $M_{\mathrm{w}}$ of 2619 and 2654 $\mathrm{Da}$, respectively, with a polydispersity $\left(M_{\mathrm{w}} / M_{\mathrm{n}}\right)$ of 1.01 . While there is a slight discrepancy between the expected $2614 \mathrm{Da}$ and observed $2619 \mathrm{Da}$, the observed MW range strongly suggests the successful formation of fluorescein-PEG-stearate with the small difference attributed to variability in the PEG chain length. Combined, these analyses confirm the successful preparation of fluorescein-PEG-stearate.

Langmuir Trough Analysis. Figure 3 characterizes the DSPC:PEG-40-stearate (9:1) monolayer at the air-water interface with the fluorescent PEG-40-stearate analogue, at 

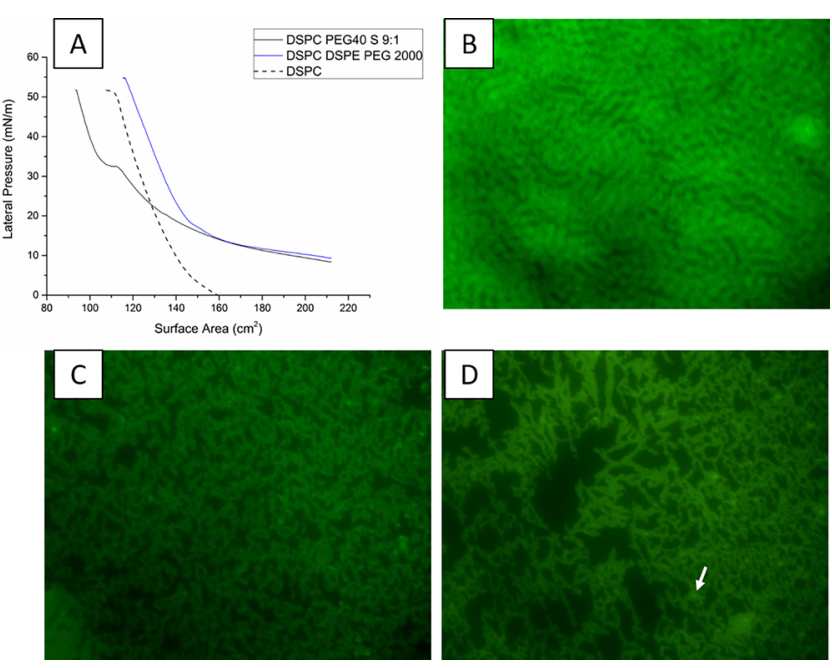

Figure 3. Langmuir trough measurements at (A) lateral pressure vs area isotherms of DSPC monolayer with and without modifications, (B) low $(\sim 10 \mathrm{mN} / \mathrm{m})$, (C) medium $(\sim 25 \mathrm{mN} / \mathrm{m})$ to (D) high lateral pressure $(\sim 40 \mathrm{mN} / \mathrm{m})$ showing the formation of domains in a mixed film of fluorescent PEG-40-stearate analogue in DSPC. Arrow indicates vesicle-like structures.

$1 \%$ concentration of PEG-40-stearate. The lateral pressure vs area $(\pi-a)$ behavior for several different lipid formulations containing DSPC lipids is shown in Figure 3A. The pure DSPC monolayer shows the well-known sublimation (gas to liquid condensed) transition at $0 \mathrm{mN} / \mathrm{m}$ where the pressure starts to rise abruptly. ${ }^{27}$ The isothermal compressibility of the monolayer can be obtained from $k_{\mathrm{T}}$ (eq 4 ).

$$
k_{\mathrm{T}}=-\frac{1}{a}\left(\frac{\partial a}{\partial \pi}\right)_{\mathrm{T}}
$$

The data indicate that the DSPC monolayer is very stiff over the entire range $(0-50 \mathrm{mN} / \mathrm{m})$ of surface pressures. For DSPC:DSPE-PEG(2000), the compressibility is similar at high pressures, but the curve is shifted to the right. The DSPC, PEG-40-stearate (9:1) monolayer, on the other hand, is qualitatively different from both DSPC alone and DSPC:DSPE-PEG(2000). A distinct region of low compressibility appears around $35 \mathrm{mN} / \mathrm{m}$. Such a plateau might be associated with a conformational transition in the components of lipid monolayers. ${ }^{11}$ However, it would then be expected to show temperature, concentration, and lipid chain length dependence. ${ }^{27}$ Previous work has shown that this is not the case for monolayers containing PEG-40-stearate ${ }^{9}$ and instead that the plateau coincides with PEG-40-stearate driven expulsion of material from the interface. The evidence of vesicular structures appearing in Figure 3D supports this explanation.

Domain-like features were observed at pressures as low as 10 $\mathrm{mN} / \mathrm{m}$ and could be clearly resolved at $25 \mathrm{mN} / \mathrm{m}$. These domains continued to grow in size on further compression, showing extensive phase separation at $40 \mathrm{mN} / \mathrm{m}$ with evidence of vesicles forming at the interface. These results are consistent with those of Lozano and Longo wherein PEG-40-stearate was found to create microbubbles less stable than those of DSPEPEG(2000). ${ }^{11}$ These also concur with the results of Carugo et al., which indicated that PEG-40-stearate and lipids are able to leave the microbubble coating and pass into the surrounding liquid. $^{19}$
Impact on Coating Lipid Order. The packing density of the lipids in the microbubble coating was analyzed by calculating the GP from spectral microscope images of Claurdan (Figure 4). GP values of microbubbles from spectral

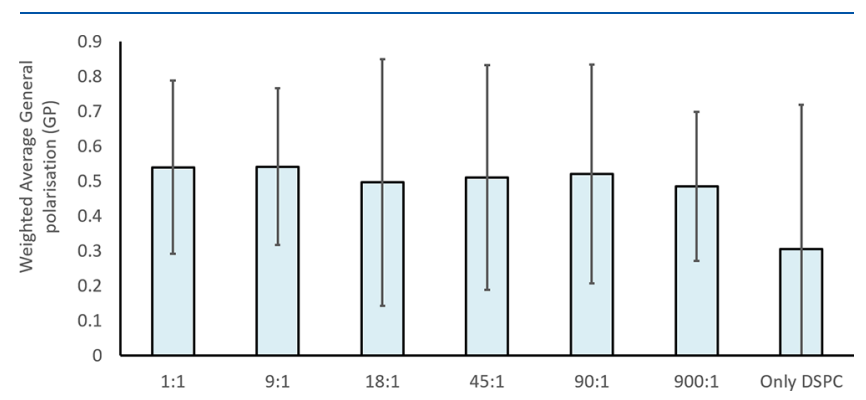

Figure 4. Weighted average generalized polarization at different ratios of DSPC to PEG-40-stearate $(n=35)$.

microscopy images were averaged and weighted to determine a mean GP for each formulation. Fluorescence microscopy images were also used to determine microbubble size and any relationship between mean GP and size. The GP values presented have not been weighted by pixel number, and values are pooled from all three repeats. Thus, GP values for microbubbles below $2 \mu \mathrm{m}$ may be more affected by noise as fewer pixels are used to calculate mean GP values.

For the range of bubble sizes investigated, increasing the concentration of PEG-40-stearate had no statistically significant effect upon the packing density of lipids in the microbubble coating. This agrees with the previous study of Hosny et al. which indicated that there was greater variability within a microbubble population than between microbubble populations. $^{20}$ It is also consistent with the suggestion in Borden et al. ${ }^{10}$ that PEG-40-stearate is excluded from the coating when the microbubble diameter is smaller than $2 \mu \mathrm{m}$, and thus, no difference in properties would be observed.

Fluorescence Microscopy Images of Bubbles. Fluorescence Microscopy with FITC-PEG-40-stearate and Dil. Images were taken of DSPC:PEG-40-stearate microbubbles that had been stained with DiI and contained 1\% PEG-40stearate-FITC (Figure 5). Intensity analysis of the confocal image stacks indicated that larger microbubbles $(>20 \mu \mathrm{m})$ had a higher intensity per pixel at $5570 \pm 141 \mathrm{AU} /$ pixel than smaller microbubbles $(<20 \mu \mathrm{m})$ at $2644 \pm 193 \mathrm{AU} /$ pixel when the midplane was examined. The opposite effect was observed with DiI; smaller microbubbles $(<20 \mu \mathrm{m})$ had an intensity per pixel of $5846 \pm 1908 \mathrm{AU} /$ pixel, whereas microbubbles larger than $20 \mu \mathrm{m}$ had an intensity per pixel of $1546 \pm 58 \mathrm{AU} /$ pixel (Supporting Information Table 1). These observations are again consistent with the exclusion of PEG-40-stearate from smaller bubbles.

A further aim of these experiments was to determine whether the presence of the emulsifier gave rise to domain formation on the surface of microbubbles within the clinical size range. It was intended that the fluorescent PEG-40stearate analogue would provide a more direct means of determining emulsifier content and bubble coating structure compared with lipid analogue dyes. No domains were observed on microbubbles with diameters smaller than $5 \mu \mathrm{m}$. The DiI images indicated that some microbubbles with diameters between 5 and $20 \mu \mathrm{m}$ did exhibit surface domains (Figure $5 \mathrm{~K}$ ), similar to those reported previously. ${ }^{11}$ The absence of a FITC signal in panel $\mathrm{J}$, however, indicates that the formation of 


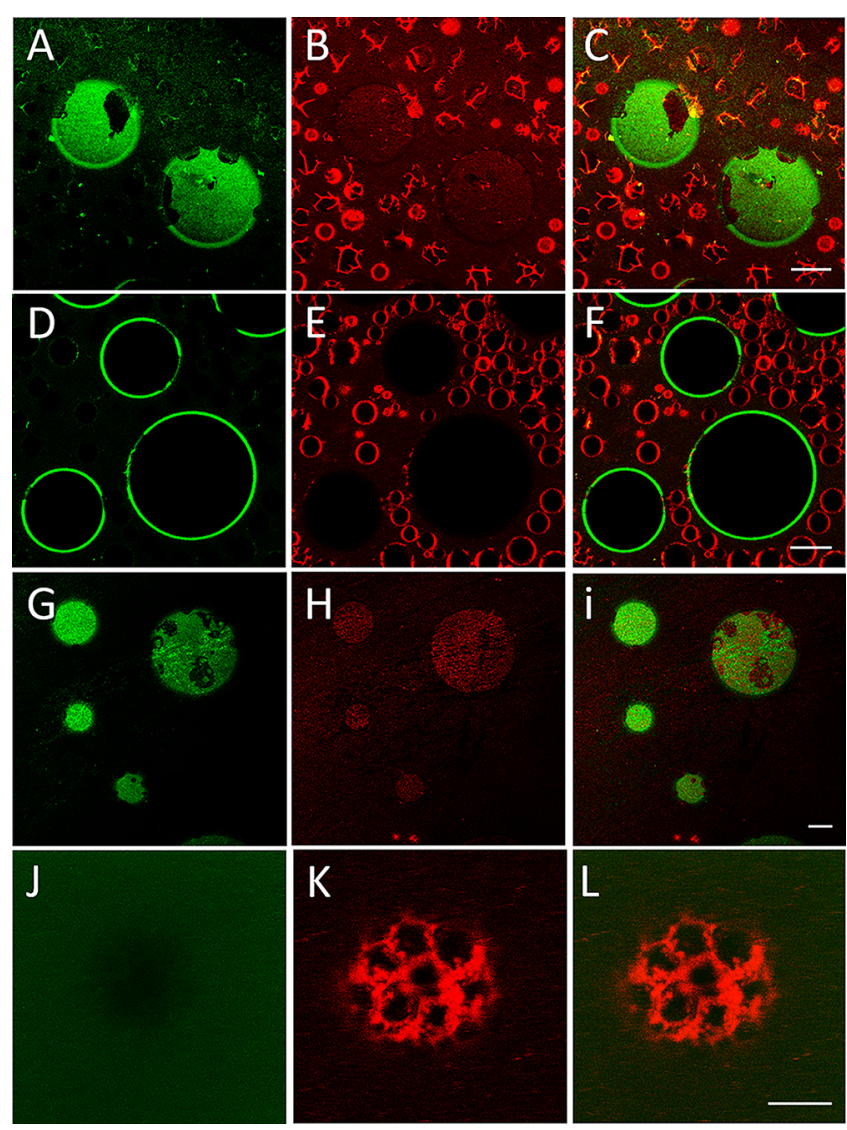

Figure 5. Images of FITC-PEG-40-stearate and DiI stained microbubbles, unwashed. First column: FITC image; second column: DiI; third column: composite. Panels A-C show two microbubbles $>20 \mu \mathrm{m}$ surrounded by smaller microbubbles with the pinhole adjusted to emphasize phase separated regions. Panels D-F show the midplanes of microbubbles with diameters from 5 to $60 \mu \mathrm{m}$ with the pinhole set to 1 airy unit. Panels G-I show microbubbles with diameters from 20 to $60 \mu \mathrm{m}$ incorporating large quantities of FITCPEG-40-stearate. $\mathrm{G}$ and $\mathrm{H}$ both appear to show domain formation, but I shows that the separated regions do not overlap. Panels J-L show an $11 \mu \mathrm{m}$ diameter microbubble with domains clearly visible in the DiI image but a negligible FITC signal also suggesting that PEG40 -stearate was not present in the domains. Scale bars: $\mathrm{C}=20 \mu \mathrm{m}, \mathrm{F}$ $=20 \mu \mathrm{m}, \mathrm{I}=20 \mu \mathrm{m}, \mathrm{L}=5 \mu \mathrm{m}$.

domains was not necessarily associated with PEG-40-stearate (this assumes that there was no energy transfer between the two dyes potentially co-localised in the membrane, i.e. from FITC-PEG40-sterate to DiI). This was further suggested by the fact that there was limited overlap in the apparent domains visible in the DiI and FITC images (Figures 5G-I).

Examining PEG-40-stearate Content within Microbubbles. To investigate further whether PEG-40-stearate was excluded from microbubbles smaller than $5 \mu \mathrm{m}$ in diameter, the microbubbles were centrifuged to remove any unincorporated PEG-40-stearate from the surrounding solution. Figures $6 \mathrm{~A}-\mathrm{C}$ indicate that the FITC-PEG-40-stearate was in fact still present in microbubbles after centrifugation. The greyscale intensity profile across the microbubble surface indicates the increase in fluorescence corresponding to the microbubble coating (Figure 6C). This was observed for all microbubbles regardless of size $(1-10 \mu \mathrm{m})$. Again, although there were variations in intensity across the microbubble coating, no domain-like features were visible on microbubbles smaller than
$5 \mu \mathrm{m}$ in diameter. This suggests that PEG-40-stearate was uniformly distributed throughout the microbubble coating and not entirely excluded by surface pressure.

Microbubbles were also created with fluorescent PEG-40 alone, without stearic acid, as a negative control. In this case, the fluorescent PEG-40 was found in solution and not in the microbubbles (Figure 6D and E), as observed for the second greyscale values (Figure 6F) after centrifugation. This suggests that PEG-40 does not passively attach to the microbubble surface and requires the stearic chain for incorporation into the microbubble coating.

Comparison of Microbubble Size and Concentration. Centrifugation of Microbubbles. DSPC-only microbubbles, DSPC:DSPE-PEG(2000) (9:1), and DSPC:PEG-40-stearate (9:1) were formulated in triplicate in PBS. These were analyzed for size and concentration, followed by centrifugation $(1000 \mathrm{rpm}, 10 \mathrm{~min})$ whereby the supernatant and infranatant were also analyzed. Centrifugation has been used to clean microbubbles and size isolate certain populations, ${ }^{28}$ and microbubble survival from centrifugation would provide information on stability. Results indicated that average size did not vary between microbubble samples within significance. However, after centrifugation, the DSPC-only microbubbles were more polydisperse relative to microbubbles formulated with DSPE-PEG(2000) and PEG-40 stearate (Figures 7A and $\mathrm{B})$. The highest concentrations (Figure $7 \mathrm{C}$ ) of microbubbles were achieved with PEG-40 stearate $\left(1 \times 10^{11}\right.$ microbubbles/ $\mathrm{mL})$, followed by DSPE-PEG $(2000)\left(1 \times 10^{10}\right.$ microbubbles/ $\mathrm{mL})$, and finally DSPC alone $\left(1 \times 10^{9}\right.$ microbubbles $\left./ \mathrm{mL}\right)$. DSPE-PEG(2000) microbubbles were more stable to centrifugation, maintaining the same average concentration as before centrifugation within experimental error, whereas PEG-40-stearate microbubbles were lower in concentration (1 $\times 10^{9}$ microbubbles $/ \mathrm{mL}$ ) after centrifugation, indicating microbubbles might be destroyed during the centrifugation process, as shown in Figure 7D. The data agree with results from Lozano et al. on the condensed phase of the lipids, resulting in increased stability when formulated with DSPE$\operatorname{PEG}(2000){ }^{11}$

Size of Vesicles. Vesicles present in the microbubble suspension were analyzed by dynamic light scattering by taking a fluid sample just before sonicating at the gas-water interface. The results shown in Figure 8 indicate that DSPC and PEG-40-stearate produces vesicles (mean diameter: 117 $\mathrm{nm}$ ) in a smaller size range than DSPC alone (mean diameter: $1500 \mathrm{~nm}$ ). DSPC and DSPE-PEG(2000) also produces smaller vesicles as well (mean diameter: $105 \mathrm{~nm}$ ). This was as expected from previous literature. ${ }^{31}$ The production of smaller vesicles could assist in the generation of the microbubble coating and the stability of the microbubble, according to the mechanism postulated by $\mathrm{Li}$ and Fogler ${ }^{32}$ whereby vesicles break up through sonication at the gas-water interface and fuse to form the microbubble coating. Hence, the size of the vesicles plays an important role as smaller vesicles will have a higher surface energy, making microbubble formation more energetically favorable. These results are also consistent with observations of phospholipid coated microbubbles via electron microscopy by Owen et al., which also indicated that the coating comprised small vesicles. ${ }^{29}$ The larger size of DSPConly vesicles might indicate that microbubbles generated from lipid fragments alone are less stable and in lower concentration, whereas microbubbles incorporating a PEG moiety contain 

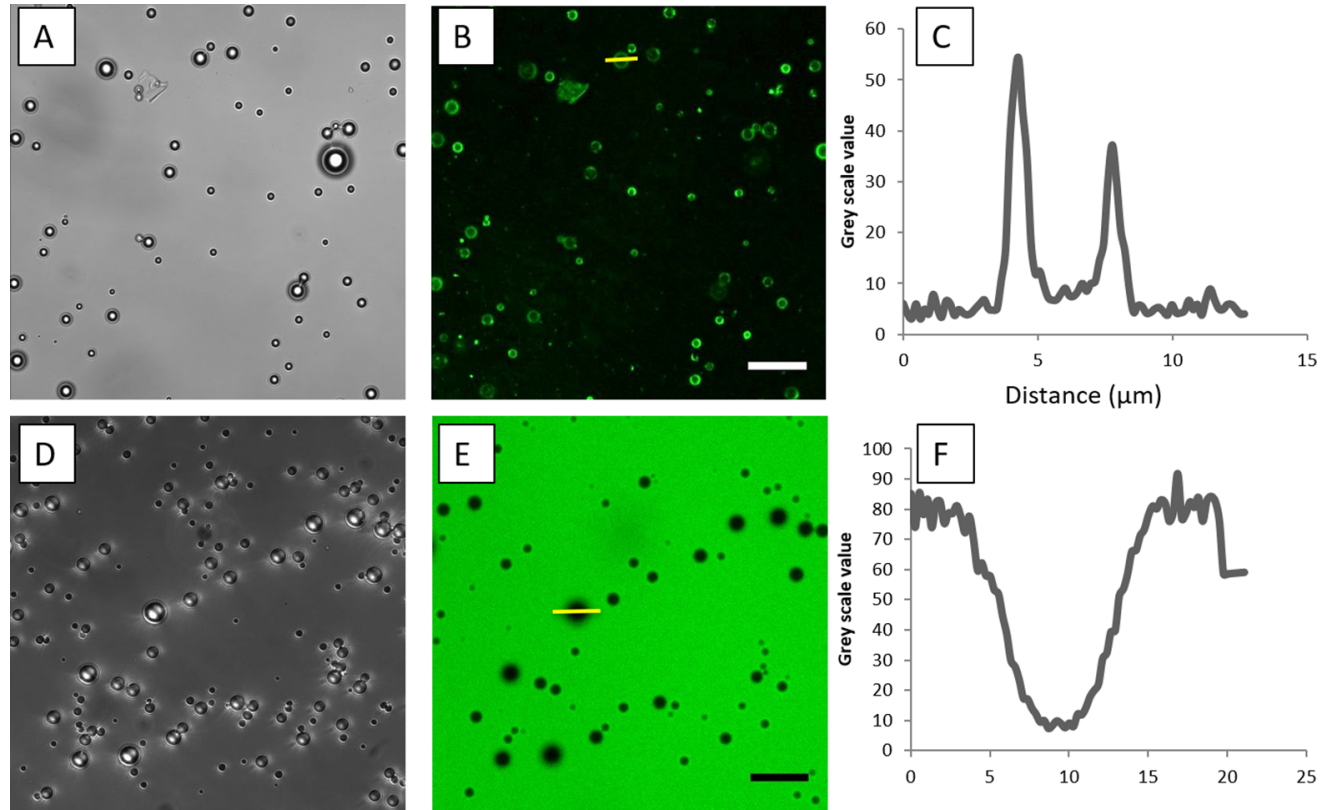

Distance $(\mu \mathrm{m})$

Figure 6. Fluorescent microbubbles. Centrifuged microbubbles created with a fluorescent PEG-40-stearate analogue in (A) brightfield and (B) fluorescence. (C) Greyscale image of fluorescence intensity drawn across a microbubble (yellow line in B). (D) Brightfield and (E) fluorescent image of microbubbles created with fluorescent PEG-40 with no attached stearic acid. (F) Greyscale image of highest fluorescence drawn across a microbubble (yellow line in E) (scale bar $20 \mu \mathrm{m}$ ).
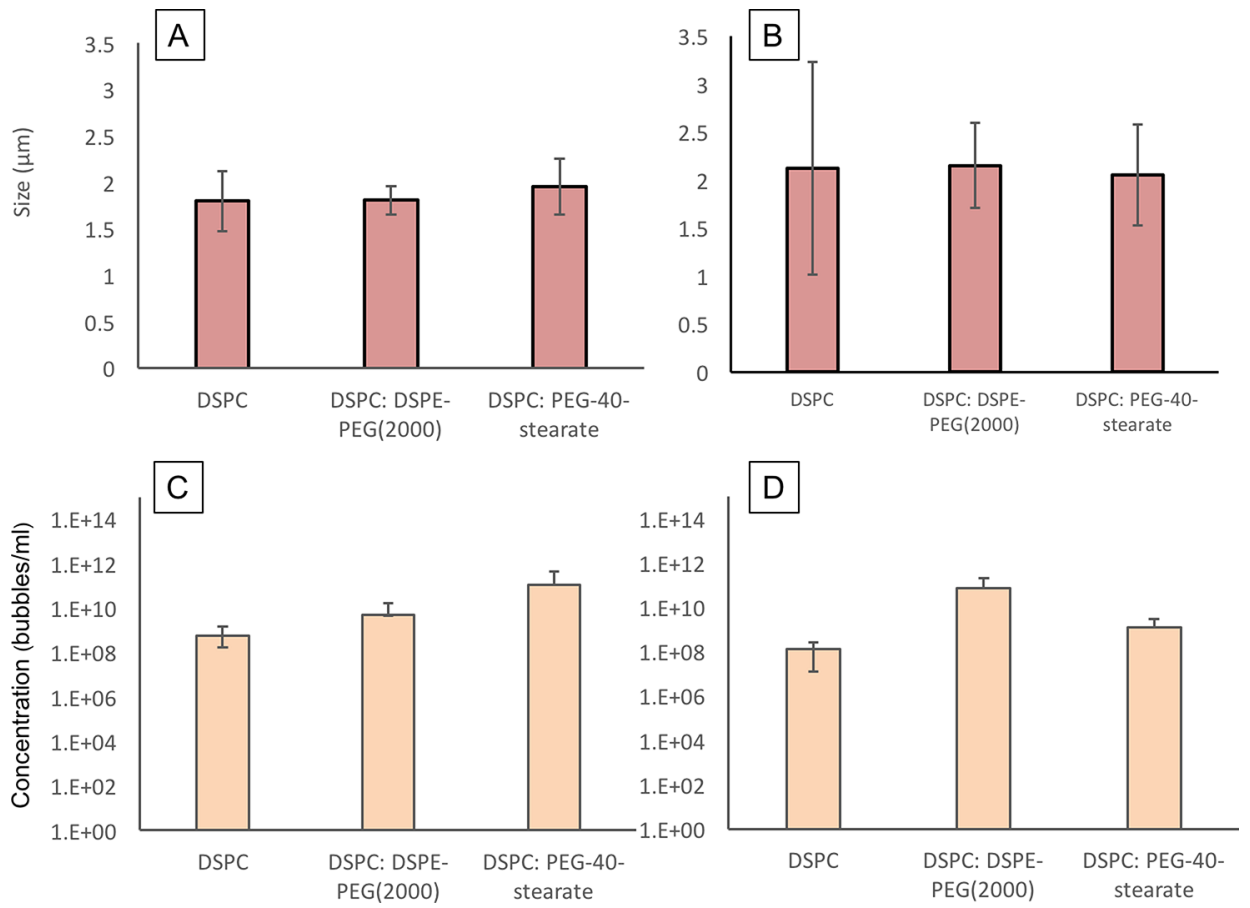

Figure 7. Average diameter and average concentration of DSPC-only microbubbles, DSPC:DSPE-PEG(2000) (9:1), and DSPC:PEG-40-stearate (9:1). (A) Size before centrifugation and (B) after centrifugation. (C) Concentration before centrifugation and (D) after centrifugation. $N=3$.

intact vesicles which have an impact on microbubble shell properties.

The role of vesicles not incorporated into the microbubble coating requires further investigation. A recent study by Fix et al. examined the clearance of PEG coated microbubbles by anti-PEG antibodies showing that microbubbles incorporating DSPE-PEG were cleared faster over time after repeated administrations by anti-PEG antibodies. ${ }^{33}$ The nanoscale vesicles present in the bubble population likely contain PEG and could have an impact on this effect.

Microbubble Serum Stability Study. DSPC:PEG-40stearate microbubbles and DSPC:DSPE-PEG(2000) microbubbles were prepared and added to human serum. At set time points, the microbubble concentration was analyzed using the Accusizer. Results indicate that over the course of $15 \mathrm{~min}$ at ambient temperature, the DSPE-PEG(2000) microbubbles 

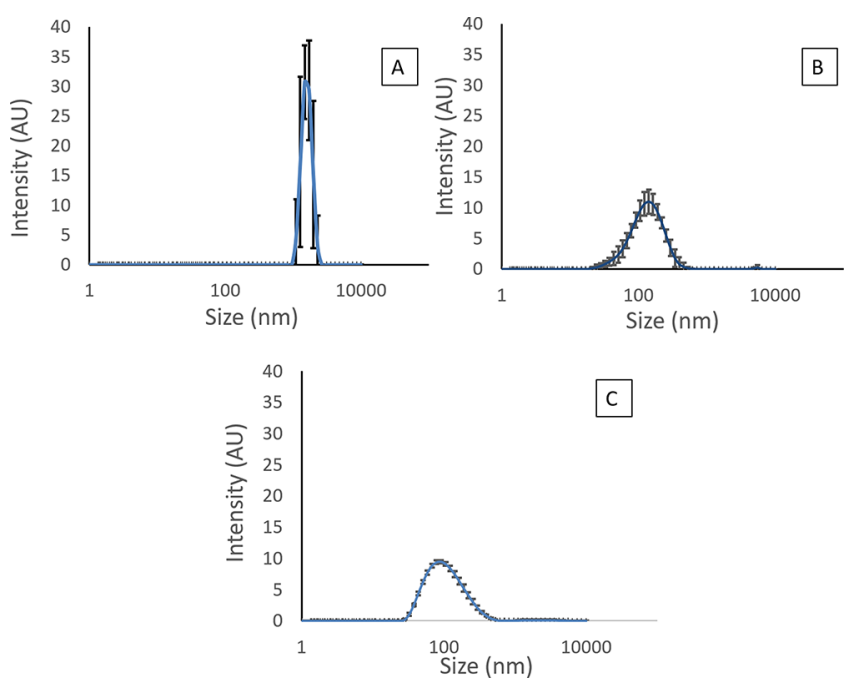

Figure 8. Dynamic light scattering results of vesicle solutions. (A) DSPC alone and (B) DSPC with PEG-40-stearate and (C) DSPC with DSPE-PEG(2000). $(n=3)$.

remained at a higher concentration than the PEG-40-stearate microbubbles (Figure 9A). This agrees with the work of
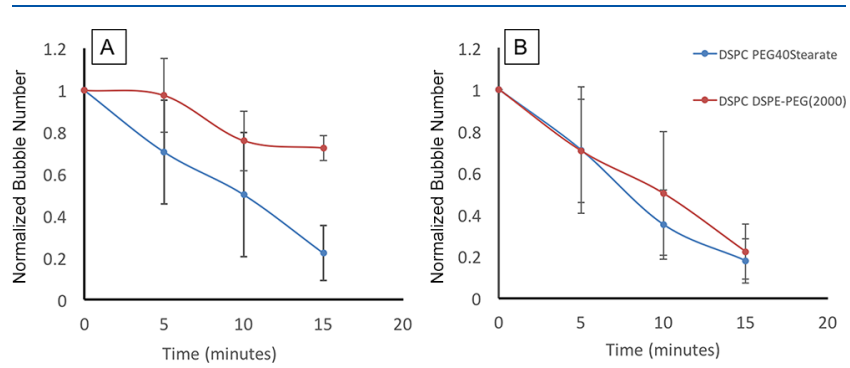

Figure 9. Serum stability of 9:1 DSPC microbubbles with PEG-40stearate and 9:1 DSPC microbubbles with DSPE-PEG(2000). (A) Bubble concentration over $15 \mathrm{~min}$ in human serum at room temperature. (B) Bubble concentration over $15 \mathrm{~min}$ in human serum at $37{ }^{\circ} \mathrm{C}(n=3)$.

Lozano et al. ${ }^{11}$ However, over the course of $10 \mathrm{~min}$, the difference in residual microbubble concentration is not statistically significant, and microbubbles incorporating DSPE-PEG(2000) only showing a marked improvement at the 15 min time point. Microbubble size and concentration were also measured over a period of 7 days from samples stored at $4{ }^{\circ} \mathrm{C}$ in a sealed vial (Supplementary Data) and showed similar trends. However, at $37{ }^{\circ} \mathrm{C}$, no difference between DSPE-PEG(2000) microbubbles and those formulated with PEG-40-stearate was observed at any time point. These differences in stability at different temperatures can be explained by comparing the isotherms of the two formulations. The isotherms for PEG-40-stearate containing monolayers show no significant change in form including the slope of the curves at all points and the pressure where the plateau appears, except that the size of the plateau shrinks. There exists a direct correspondence between the monolayer and bilayer surface pressure, and under equilibrium, the two are equal. ${ }^{30-32}$ While the surface pressure for a bubble cannot be ascertained without a direct measurement, it can still be deduced from the isotherms that the compressibility of the film remains similar for all pressures when increasing the temperature from 18 to $37{ }^{\circ} \mathrm{C}$. In contrast, DSPE-PEG2000 containing films become more compressible with increasing temperature. ${ }^{33}$ Statistical thermodynamics provide a direct relationship between lipid film permeability and its compressibility, ${ }^{34,35}$ and thus, the gas permeability of PEG40S bubbles expected to remain the same while it is expected to increase for DSPE-PEG2000 bubbles as the temperature is increased from 18 to $37^{\circ} \mathrm{C}$, leading to a reduction in microbubble stability.

It is important to note, however, that "stability" in the context of clinical applications is also determined by lung and liver capture, and further work is required to compare the in vivo circulatory stability of the two formulations.

Backscatter Acoustic Intensity Comparison of Microbubbles. The average backscatter intensity of 9:1 DSPC microbubbles with PEG-40-stearate and 9:1 DSPC microbubbles with DSPE-PEG(2000) was compared as shown in Figure 10. No significant difference was seen between the two
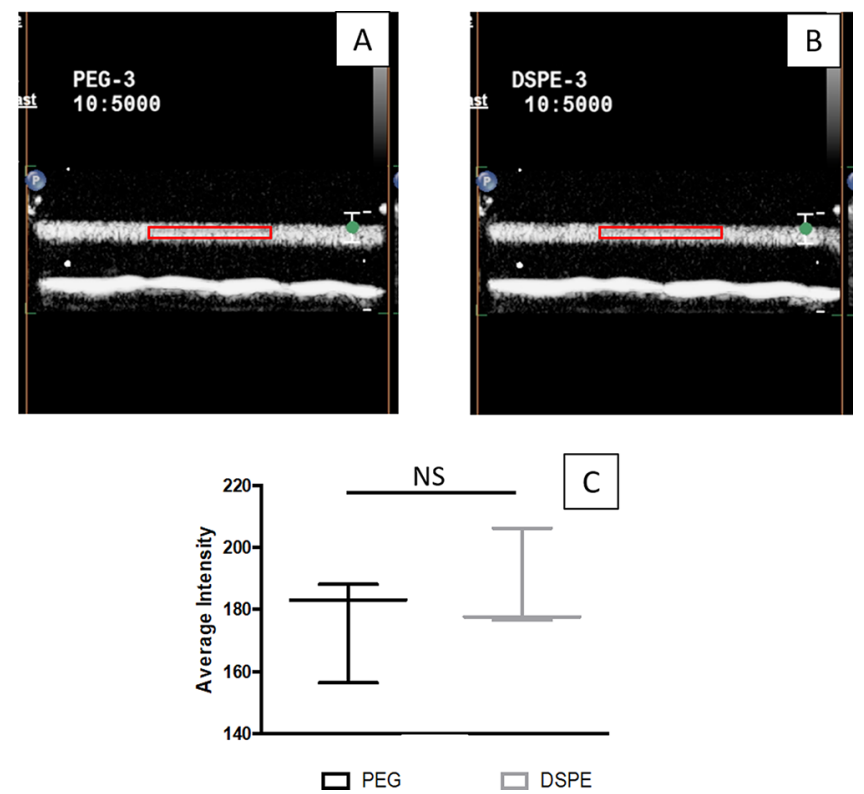

Figure 10. Acoustic intensity of 9:1 DSPC microbubbles with PEG40-stearate and 9:1 DSPC microbubbles with DSPE-PEG(2000). (A) Representative (contrast mode) image of 9:1 DSPC microbubbles with PEG-40-stearate in the flow phantom and (B) representative (contrast mode) image of 9:1 DSPC microbubbles with DSPEPEG(2000) in the flow phantom. Red box is the region of interest used to quantify the backscatter intensity for each image. (C) Average intensity of two microbubble populations. Significance was measured using Student's one-tailed $t$-test $(n=3)$.

microbubble formulations $(p=0.3719)$, indicating that both types of bubble would behave similarly as ultrasound contrast agents.

\section{CONCLUSIONS}

A fluorescent form of PEG-40-stearate was successfully synthesized and used to determine the location of the PEG40-stearate molecule within a lipid microbubble coating. Langmuir trough measurements revealed that PEG-40-stearate changes the isotherm of DSPC alone, and images of the fluorescent PEG-40-stearate analogue indicated domain formation and vesicles entering the solution and leaving the lipid film at $30 \mathrm{mN} / \mathrm{m}$ in contrast to films of DSPEPEG(2000) and DSPC alone, in which coating integrity was maintained. 
Spectral imaging showed that PEG-40-stearate did not have a statistically significant impact on lipid order in the bubble coating at any concentration. This indicates that PEG-40stearate likely enhances microbubble formation without impacting shell properties and that most PEG-40-stearate is excluded from the coating and enters the solution. Fluorescence microscopy, however, revealed that some PEG40-stearate does remain within the bubble coating.

Staining with DiI allowed observation of domains as previously reported by Borden et al. ${ }^{9}$ In the present study, however, domains were also only observed on larger microbubbles $(>5 \mu \mathrm{m})$. In addition, the proportion PEG-40stearate was higher in microbubbles with diameters $>8 \mu \mathrm{m}$.

No differences in microbubble size or concentration were found relative to DSPE-PEG(2000) formulated microbubbles, and both PEG-40-stearate and DSPE-PEG(2000) were stable to centrifugation. Both formulations created $\sim 100 \mathrm{~nm}$ vesicles, indicating similar microbubble formation mechanisms.

At room temperature, DSPE-PEG(2000) microbubbles maintained a higher concentration for a longer period of time in human serum than PEG-40-stearate microbubbles. However, at $37{ }^{\circ} \mathrm{C}$, no statistically significant difference could be discerned. Furthermore, no statistical difference was found in acoustic intensity between the two microbubble populations in a flow phantom at $37^{\circ} \mathrm{C}$.

\section{ASSOCIATED CONTENT}

\section{S Supporting Information}

The Supporting Information is available free of charge on the ACS Publications website at DOI: 10.1021/acs.langmuir.8b02516.

Intensity/pixel of FITC and DiI for microbubbles of different diameters, microbubble diameter as a function of time when stored in a sealed vial at $4{ }^{\circ} \mathrm{C}$, and microbubble concentration as a function of time when stored in a sealed vial at $4{ }^{\circ} \mathrm{C}(\mathrm{PDF})$

\section{AUTHOR INFORMATION}

\section{Corresponding Author}

*E-mail: eleanor.stride@eng.ox.ac.uk.

\section{ORCID $\odot$}

Shamit Shrivastava: 0000-0003-0916-7336

Eleanor Stride: 0000-0003-3371-5929

Notes

The authors declare no competing financial interest.

\section{ACKNOWLEDGMENTS}

The authors thank the Engineering and Physical Sciences Research Council for funding the work through grants EP/ I021795/1 and EP/L024012/1. E.S. thanks the Institute of Engineering and Technology for support through the AF Harvey prize. J.F.C. thanks Norbrook Laboratories Ltd. for funding an endowed chair. All experimental data are available through the University of Oxford ORA data repository at 10.5287/bodleian:PVMVN90da.

\section{REFERENCES}

(1) Quaia, E. Contrast Media in Ultrasonography. Medical Radiology 2005, 117

(2) Stride, E.; Edirisinghe, M. Novel microbubble preparation technologies. Soft Matter 2008, 4 (12), 2350.
(3) Lindner, J.R.; Kaul, S. Delivery of drugs with ultrasound. Echocardiography 2001, 18 (4), 329-337.

(4) McEwan, C.; et al. Oxygen carrying microbubbles for enhanced sonodynamic therapy of hypoxic tumours. J. Controlled Release 2015, 203, 51-56.

(5) Owen, J. Reducing tumour hypoxia via oral administration of oxygen nanobubbles. PLoS One 2016, 11 (12), e0168088.

(6) Spahn, D. R. Blood substitutes. Critical Care 1999, 3 (5), R91R92.

(7) Ferrara, K. W.; Borden, M. A.; Zhang, H. Lipid-Shelled Vehicles: Engineering for Ultrasound Molecular Imaging and Drug Delivery. Acc. Chem. Res. 2009, 42 (7), 881-892.

(8) Kim, D. H.; et al. Mechanical properties and microstructure of polycrystalline phospholipid monolayer shells: Novel solid microparticles. Langmuir 2003, 19 (20), 8455-8466.

(9) Borden, M. A.; et al. Surface phase behavior and microstructure of lipid/PEG-emulsifier monolayer-coated microbubbles. Colloids Surf., B 2004, 35 (3-4), 209-223.

(10) Borden, M. A.; et al. Lateral phase separation in lipid-coated microbubbles. Langmuir 2006, 22 (9), 4291-4297.

(11) Lozano, M. M.; Longo, M. L. Microbubbles Coated with Disaturated Lipids and DSPE-PEG2000: Phase Behavior, Collapse Transitions, and Permeability. Langmuir 2009, 25 (6), 3705-3712.

(12) Gerber, F.; et al. Fluidization of a dipalmitoyl phosphatidylcholine monolayer by fluorocarbon gases: Potential use in lung surfactant therapy. Biophys. J. 2006, 90 (9), 3184-3192.

(13) Venegas, B.; et al. High Vapor Pressure Perfluorocarbons Cause Vesicle Fusion and Changes in Membrane Packing. Biophys. J. 2008, 95 (10), 4737-4747.

(14) Fainerman, V. B.; Aksenenko, E. V.; Miller, R. Influence of alkane and perfluorocarbon vapors on adsorbed surface layers and spread insoluble monolayers of surfactants, proteins and lipids. $A d v$. Colloid Interface Sci. 2017, 244, 100-112.

(15) Kooiman, K. DSPC or DPPC as main shell component influences ligand distribution and binding area of lipid-coated targeted microbubbles. Eur. J. Lipid Sci. Technol. 2014, 116, 1217.

(16) Abou-Saleh, R. H.; et al. Poly(ethylene glycol) Lipid-Shelled Microbubbles: Abundance, Stability, and Mechanical Properties. Langmuir 2014, 30 (19), 5557-5563.

(17) Shih, R.; Lee, A. P. Post-Formation Shrinkage and Stabilization of Microfluidic Bubbles in Lipid Solution. Langmuir 2016, 32 (8), $1939-1946$.

(18) Segers, T.; et al. Stability of Monodisperse PhospholipidCoated Microbubbles Formed by Flow-Focusing at High Production Rates. Langmuir 2016, 32 (16), 3937-3944.

(19) Carugo, D.; et al. Modulation of the molecular arrangement in artificial and biological membranes by phospholipid-shelled microbubbles. Biomaterials 2017, 113, 105-117.

(20) Hosny, N. A.; et al. Mapping microbubble viscosity using fluorescence lifetime imaging of molecular rotors. Proc. Natl. Acad. Sci. U. S. A. 2013, 110 (23), 9225-9230.

(21) Aron, M.; et al. Spectral imaging toolbox: Segmentation, hyperstack reconstruction, and batch processing of spectral images for the determination of cell and model membrane lipid order. BMC Bioinf. 2017, 18, 254.

(22) Parasassi, T.; et al. Cholesterol modifies water concentration and dynamics in phospholipid bilayers: a fluorescence study using Laurdan probe. Biophys. J. 1994, 66 (3 Part 1), 763-768.

(23) Sennoga, C. A.; et al. On sizing and counting of microbubbles using optical microscopy. Ultrasound in Medicine and Biology 2010, 36 (12), 2093-2096.

(24) Borden, M. A.; et al. Ultrasound radiation force modulates ligand availability on targeted contrast agents. Mol. Imaging 2006, 5 (3), 139-147.

(25) Quaia, E. Microbubble ultrasound contrast agents: An update. European Radiology 2007, 17 (8), 1995-2008.

(26) Mannaris, C.; et al. Gas-Stabilizing Gold Nanocones for Acoustically Mediated Drug Delivery. Adv. Healthcare Mater. 2018, 7 (12), 1800184. 
(27) Albrecht, O.; Gruler, H.; Sackmann, E. Polymorphism of phospholipid monolayers. J. Phys. (Paris) 1978, 39 (3), 301-313.

(28) Feshitan, J. A.; et al. Microbubble size isolation by differential centrifugation. J. Colloid Interface Sci. 2009, 329 (2), 316-324.

(29) Owen, J.; Stride, E. Technique for the Characterization of Phospholipid Microbubbles Coatings by Transmission Electron Microscopy. Ultrasound in Medicine and Biology 2015, 41 (12), $3253-3258$.

(30) Feng, S.-s. Interpretation of Mechanochemical Properties of Lipid Bilayer Vesicles from the Equation of State or Pressure-Area Measurement of the Monolayer at the Air-Water or Oil-Water Interface. Langmuir 1999, 15 (4), 998-1010.

(31) Jähnig, F. What is the surface tension of a lipid bilayer membrane? Biophys. J. 1996, 71 (3), 1348-1349.

(32) MacDonald, R. C.; Simon, S. A. Lipid monolayer states and their relationships to bilayers. Proc. Natl. Acad. Sci. U. S. A. 1987, 84 (12), 4089-4093.

(33) Chou, T.-H.; Chu, I. M. Thermodynamic characteristics of DSPC/DSPE-PEG2000 mixed monolayers on the water subphase at different temperatures. Colloids Surf., B 2003, 27 (4), 333-344.

(34) Nagle, J. F.; Scott, H. L. Lateral compressibility of lipid monoand bilayers. Theory of membrane permeability. Biochim. Biophys. Acta, Biomembr. 1978, 513 (2), 236-243.

(35) Wunderlich, B.; et al. Phase-State Dependent Current Fluctuations in Pure Lipid Membranes. Biophys. J. 2009, 96 (11), $4592-4597$. 